\title{
Recuperación de la Tasa de Emisión de una Fuente Contaminante: Análisis de la Existencia, la Unicidad y la Estabilidad de las Soluciones
}

\author{
David Parra-Guevara y Yuri N. Skiba \\ Centro de Ciencias de la Atmósfera, Universidad Nacional Autónoma de México, Circuito Exterior, Ciudad \\ Universitaria, México, D. F., C. P. 04510, México (Email: pdavid@atmosfera.unam.mx; skiba@unam.mx)
}

Recibido Nov. 26, 2015; Aceptado Feb. 4, 2016; Versión final Mar. 31, 2016, Publicado Oct. 2016

\begin{abstract}
Resumen
En este trabajo se analiza la existencia, la unicidad y la estabilidad de las soluciones en el problema de la recuperación de la tasa de emisión de una fuente puntual no-estacionaria a partir de datos de concentración de contaminantes que contienen una perturbación de pequeña amplitud. Se presentan ejemplos numéricos que muestran la inestabilidad en el problema inverso y permiten identificar el tipo de perturbación en los datos que es más significativa para este problema. La ecuación de balance de materia para un contaminante se utiliza para analizar la existencia de soluciones. A través de las funciones adjuntas y el principio de dualidad para la anomalía de la concentración puntual, se prueba que el problema inverso siempre es inestable cuando el modelo de dispersión tiene asociado un modelo adjunto cuya solución es de clase $C^{1}$. Se analiza la existencia y la unicidad de la tasa de emisión para esta formulación del problema inverso. Se concluye que es indispensable introducir una regularización en el problema inverso que filtre los errores de alta frecuencia en los datos y permita obtener una estimación adecuada de la tasa de emisión.
\end{abstract}

\section{Recovery of the Emission Rate of a Pollution Source: Analysis of the Existence, Uniqueness and Stability of Solutions}

\begin{abstract}
In this work the existence, uniqueness and stability of the solutions in the problem of recovery of the emission rate of a non-steady point source from concentration data of pollutants with a perturbation of small amplitude, are analyzed. Numerical examples that demonstrate the instability of the inverse problem and identify the type of perturbation in the data that is most meaningful to this problem are presented. The mass balance equation for a pollutant is used to analyze the existence of solutions. Through the adjoint functions and the duality principle for the anomaly of the punctual concentration, it is shown that the inverse problem is always unstable when the dispersion model has an associated adjoint model whose solution is of class $C^{1}$. The existence and uniqueness of the emission rate for this formulation of the inverse problem is analyzed. It is concluded that it is indispensable to introduce a regularization in the inverse problem to filter high frequency errors in the data and to obtain an adequate estimate of the emission rate.
\end{abstract}




\section{INTRODUCCIÓN}

La estimación de los parámetros que caracterizan a las fuentes puntuales con base en datos de la concentración de contaminantes es un problema inverso que aparece en el estudio de la contaminación en el océano, la atmósfera y el agua subterránea (Sharma et al., 2014; Lushi y Stockie, 2010; Skaggs y Kabala, 1994). La solución de este problema es relevante para establecer el grado de responsabilidad entre los potenciales agentes involucrados en la contaminación local y regional, la estimación del impacto ambiental, así como la definición de acciones para proteger a la población asentada en zonas contaminadas. Algunos ejemplos importantes de la estimación de parámetros incluyen la evaluación de la tasa de descarga de sustancias radioactivas en el océano (Sharma et al., 2014), y la estimación de la intensidad y la ubicación de pruebas 0 accidentes nucleares con base en observaciones de sustancias radioactivas en la atmósfera (Seibert et al., 2002; Pudykiewicz, 1998).

El estudio sistemático de este problema con el fin de estimar la intensidad y el sitio de las fuentes y sumideros de las trazas que constituyen la atmósfera, o de las fuentes de emisión de contaminantes, es relativamente nuevo. En particular, en la última década diferentes técnicas de modelación inversa se han utilizado para estimar la tasa de emisión de las fuentes contaminantes y corregir los inventarios de emisiones (Brioude et al., 2011; Lushi y Stockie, 2010). Sin embargo, el reconocimiento de la inestabilidad, característica inherente en este tipo de problema inverso, aparece ya mencionado en un estudio global sobre fuentes y sumideros de $\mathrm{CO}_{2}$ (Bolin y Keeling, 1963).

Diferentes autores señalan que las principales dificultades en este problema inverso son la ausencia de funciones de emisión compatibles con el proceso de transporte en la atmósfera y los datos de concentración, y la catastrófica inestabilidad que surge por la presencia de cierta clase de error en los datos (Enting y Newsam, 1990). Tales errores son consecuencia de la incertidumbre en la medición que introduce el equipo de monitoreo, los errores sistemáticos en el proceso de muestreo y los errores de aproximación en la salida de los modelos de dispersión de contaminantes, entre otros. Los datos útiles para la estimación de parámetros pueden contener una combinación de dichos errores. Esto se debe a que el proceso de inversión necesita información de la anomalía de la concentración producida por las fuentes de emisión (Parra-Guevara et al., 2015), lo cual requiere restar datos de diferente procedencia (salida de modelos de dispersión y datos que provienen de instrumentos de medición).

Dos argumentos se utilizan frecuentemente para mostrar la inestabilidad en casos particulares de este tipo de problema inverso (Enting y Newsam, 1990). El primero consiste en formular el problema como un sistema lineal $A x=y$, donde el vector $y$ agrupa los datos de concentración, el vector $x$ contiene los parámetros de las fuentes y la matriz $A$ es la matriz de transferencia (dispersión). La demostración de la inestabilidad consiste en probar que la matriz $A$ presenta valores singulares cercanos a cero, lo cual amplifica los errores en los datos durante la estimación de $x$ por un factor que es precisamente el inverso de dichos valores singulares. La desventaja de este argumento es que es necesario discretizar el problema directo continuo para obtener la matriz de transferencia, y es bien sabido que tal proceso puede inducir inestabilidad aun cuando un modelo (continuo) esté bien formulado. El segundo argumento consiste en mostrar que el operador $\mathfrak{I}$ del problema directo continuo es compacto, y por lo tanto, el operador inverso $\mathfrak{J}^{-1}$ es discontinuo (no-acotado). Por lo que pequeñas variaciones en los datos de concentración pueden generar grandes cambios en los parámetros de las fuentes. La desventaja de tal prueba es que ésta no exhibe explícitamente la inestabilidad, o su comportamiento bajo diferentes tipos de errores en los datos.

En este trabajo se analiza la existencia, la unicidad y la estabilidad de las soluciones en el problema de la recuperación de la tasa de emisión de una fuente puntual no-estacionaria cuando los datos de concentración contienen errores de pequeña amplitud. Utilizamos la ecuación de balance de masa de un modelo de dispersión tridimensional bien formulado para mostrar que hay conjuntos de datos de concentración, muy cercanos a los datos exactos y con la misma suavidad de estos, con los cuales es imposible recuperar una tasa de emisión. Posteriormente, demostramos la inestabilidad del problema inverso usando funciones adjuntas y un principio de dualidad para estimar la concentración puntual de un contaminante. Esta prueba exhibe explícitamente el error en la tasa de emisión recuperada como función del error en los datos de concentración. Con esta formulación general se analiza la existencia y la unicidad de la tasa de emisión. Finalmente, se propone una regularización para resolver en forma adecuada el problema inverso.

Es importante notar que aún cuando existen métodos para el análisis de datos de la concentración de contaminantes, como el de Factor de Enriquecimiento (Rojano et al., 2014), los cuales permiten diferenciar (cualitativamente) entre contaminantes procedentes de fuentes antropogénicas y naturales, es de suma importancia estimar cuantitativamente la tasa de emisión de una sustancia con el fin de reconstruir el impacto regional que tiene una fuente contaminante. Dicha reconstrucción se obtiene a través de la 
incorporación de la tasa de emisión a un modelo de la calidad del aire. Ya que la dispersión de los contaminantes en la atmósfera es un fenómeno complejo (Vázquez et al., 2012; Enting y Newsam, 1990), el uso de modelos matemáticos es indispensable para el pronóstico de la concentración de diferentes contaminantes, el análisis de estrategias de control de emisiones, y para la estimación de los parámetros de dispersión y de las fuentes de emisión. En este trabajo utilizamos tres modelos con diferente grado de sofisticación para analizar el problema de la recuperación de la tasa de emisión de una fuente contaminante. La importancia del estudio radica en la conclusión que es común en los tres casos: hay inestabilidad en el proceso de inversión de datos, y por lo tanto, hay dificultad en la obtención de una tasa de emisión compatible con los datos de concentración de contaminantes y el proceso de dispersión en la atmósfera. Alertamos al lector sobre este comportamiento porque dicha dificultad no se corrige realizando un esfuerzo o gasto adicional para incorporar al proceso de inversión más datos de concentración procedentes de otras estaciones de monitoreo o por medio de un muestreo en intervalos de tiempo más cortos, lo cual, como se muestra en el análisis de los modelos de este trabajo, tiende a amplificar el error en la estimación de la tasa de emisión. La estimación adecuada de la tasa de emisión requiere la introducción de una regularización en el problema inverso que filtre los errores responsables de la inestabilidad (ParraGuevara et al., 2015; Seibert et al., 2002; Enting y Newsam, 1990), es decir, se necesita de un procesamiento de los datos a través de consideraciones matemáticas adicionales para obtener una solución satisfactoria. Al final de este trabajo formulamos una regularización de este tipo, en la cual minimizamos un funcional que filtra los errores de alta frecuencia en los datos. Los argumentos matemáticos contenidos en el trabajo son originales y se han preparado con el fin de presentar claramente los aspectos más delicados del problema inverso. En particular, la formulación adjunta que se introduce como parte del argumento de análisis general permite estudiar de manera sencilla el problema de unicidad de las soluciones y proponer la regularización del problema inverso como un problema variacional que se resuelve de forma eficiente.

\section{INESTABILIDAD DEL PROBLEMA INVERSO SIMPLIFICADO}

En esta sección se considera un modelo simplificado (modelo de dispersión de dimensión cero) con el fin de identificar el tipo de perturbación en los datos de concentración que hace explícita la inestabilidad en el problema inverso. Se asume que la anomalía de la concentración del contaminante $\phi$ satisface las siguientes ecuaciones (Parra-Guevara y Skiba, 2003).

$$
(\phi)_{t}+\left(\sigma+V^{-1} u a^{2}\right) \phi=V^{-1} Q(t), \quad 0<t<T ; \quad \phi(0)=0
$$

Aquí se supone que, en todo momento, el contaminante forma una mezcla homogénea con el aire en la región $\mathbf{D}=[0, a] \times[0, a] \times[0, a]$, la cual es un cubo con arista de longitud a y volumen $V=a^{3}$. La velocidad del viento $u$ se asume de tipo estacionario, con dirección perpendicular a una pared vertical del cubo. Finalmente, $\sigma$ denota el coeficiente de transformación química del contaminante. Con estas simplificaciones, la ecuación (1) es un caso particular de la ecuación de balance de masa del modelo de dispersión que se presenta en la siguiente sección, para un sólo contaminante, sin sedimentación y considerando una fuente puntual en la región $\mathbf{D}$ con tasa de emisión $Q(t)$.

Para mostrar la inestabilidad del problema inverso se calcula $Q(t)$ directamente de la ecuación (1). Usando fórmulas de diferencias finitas centradas de segundo orden de aproximación se obtiene el modelo discreto: $\left(\phi_{j+1}-\phi_{j-1}\right) /(2 \Delta t)+0.5 r\left(\phi_{j+1}+\phi_{j-1}\right)=V^{-1} Q_{j}$, donde $r=\sigma+V^{-1} u a^{2}$. También, $Q_{j}$ aproxima a $Q\left(t_{j}\right)$ en los momentos $t_{j}=j \Delta t, j=0,1, \ldots, L, \Delta t$ es la longitud de tiempo entre los datos y $\phi_{j}$ es la anomalía de la concentración del contaminante en el instante $t_{j}$. Así, se obtiene el siguiente esquema para recuperar $Q(t)$ :

$$
Q_{j}=0.5 V\left\{(r+1 / \Delta t) \phi_{j+1}+(r-1 / \Delta t) \phi_{j-1}\right\}, \quad j=1, \ldots, L
$$

donde se considera que $\phi_{0}=0$. La serie de tiempo sintética que utilizamos para probar el esquema (2) se construye con base al siguiente par de funciones que satisfacen el modelo de dispersión (1):

$$
\begin{aligned}
& Q(t)=\{0, \quad 0 \leq t<2 ; \quad q, \quad 2 \leq t \leq 4 ; \quad 0, \quad 4<t \leq 10\} \\
& \phi(t)=\left\{0, \quad 0 \leq t<2 ; \quad q(r V)^{-1}\left(1-e^{-r(t-2)}\right), \quad 2 \leq t \leq 4 ; \quad q(r V)^{-1}\left(e^{-r(t-4)}-e^{-r(t-2)}\right), \quad 4<t \leq 10\right\}
\end{aligned}
$$

donde los valores de los parámetros son los siguientes: $a=1 \mathrm{~km}, u=0.5 \mathrm{kmh}^{-1}, \quad \sigma=0.0001 h^{-1}, T=10 h$ y $q=100 \mathrm{kgh}^{-1}$. Considerando la función $\phi(t)$ en (3), la serie de tiempo sintética se genera como: $\phi_{j}=\phi\left(t_{j}\right)+\delta \phi_{j}$, $t_{j}=j \Delta t, j=1, \ldots, L$, donde los valores aleatorios $\delta \phi_{j}$ están uniformemente distribuidos en el intervalo $(-0.5,0.5)$ y la amplitud de los errores está normalizada por el $15 \%$ del valor máximo de $\phi$. La Figura 1 muestra un 
ejemplo de la serie de tiempo sintética construida para $\Delta t=0.05 \mathrm{~h}$. La Figura 2 muestra la tasa de emisión $Q_{\varepsilon}(t)$ que se obtiene del esquema (2) a partir de dicha serie de tiempo.

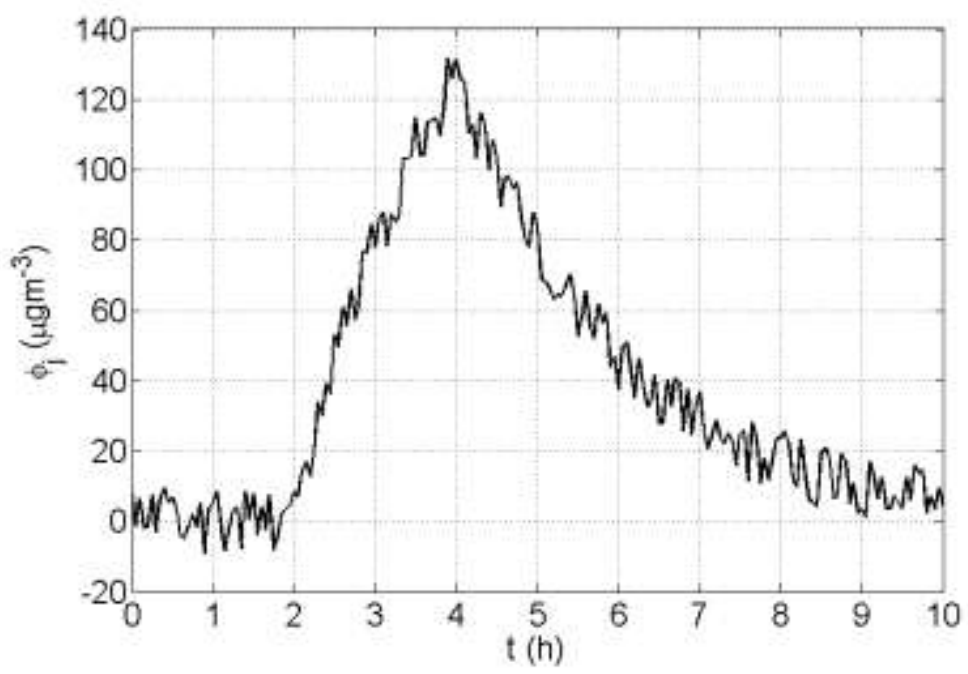

Fig. 1: Serie de tiempo sintética: $\phi_{j}=\phi\left(t_{j}\right)+\delta \phi_{j}$.

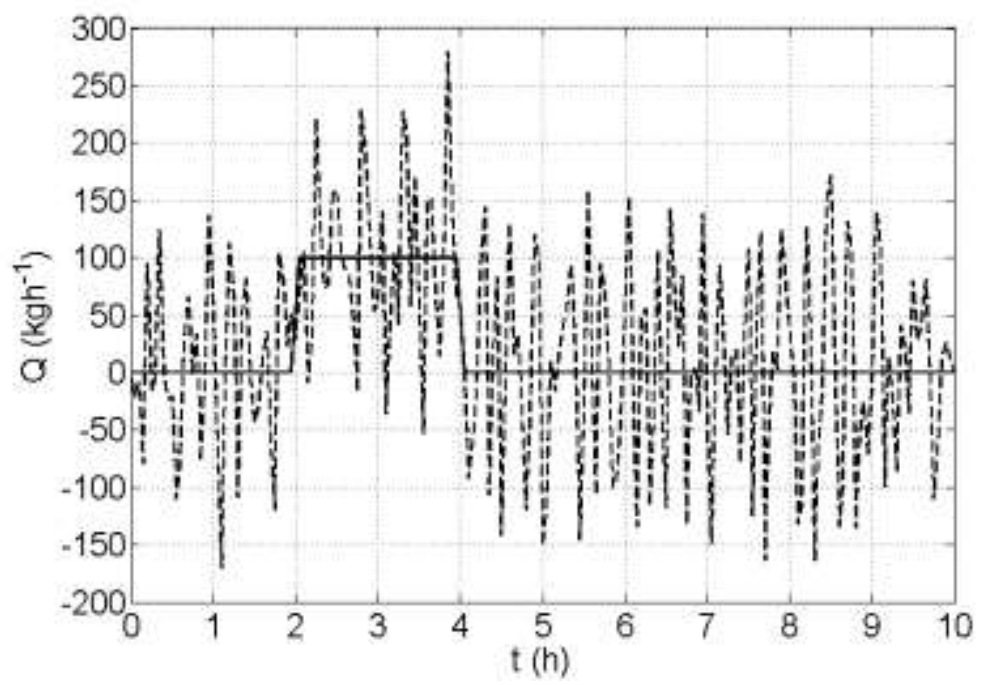

Fig. 2: Línea punteada: $Q_{\varepsilon}(t)$ obtenida con (2), línea continua: $Q(t)$ definida por (3).

Se nota que la tasa real $Q(t)$ no se calcula en forma adecuada ya que el error de aproximación ha aumentado hasta $175 \%$ en algunos instantes. Explicaremos este fenómeno de inestabilidad en forma general. Substituyendo $\phi_{j}=\phi\left(t_{j}\right)+\delta \phi_{j}$ en la ecuación (2) se obtiene la siguiente estimación:

$\left|Q\left(t_{j}\right)-Q_{j}\right| \leq r V \max _{j}\left|\delta \phi_{j}\right|+V\left|\left(\delta \phi_{j+1}-\delta \phi_{j-1}\right) / 2 \Delta t\right|+o\left((\Delta t)^{2}\right)$

Así, el error de aproximación en la tasa de emisión se incrementa debido a la amplitud de la perturbación en los datos y a la derivada de dicha perturbación. En particular, si $\delta \phi(t)=\varepsilon(t) \operatorname{sen}(4 \omega \pi t / T),|\varepsilon(t)|<\varepsilon 0<<1$, entonces $\delta^{\prime} \phi(T / 2)=\varepsilon(T / 2)(4 \omega \pi / T) \rightarrow \infty$, cuando $\omega \rightarrow \infty$. Lo cual provoca el crecimiento del error de acuerdo a (4). Esto sugiere que las perturbaciones de alta frecuencia en los datos son ideales para exhibir la inestabilidad del problema inverso. Note que el error aleatorio contenido en un gran número de datos de concentración registrados dentro del intervalo $(0, T)$ forman una perturbación de alta frecuencia.

\section{MODELO DE DISPERSIÓN TRIDIMENSIONAL}

Iniciamos el análisis de existencia de soluciones en el problema inverso describiendo brevemente un modelo que es posible utilizar para predecir la dispersión de varias sustancias emitidas desde fuentes puntuales. Los detalles pueden consultarse en Parra-Guevara et al. (2010). Sea $\mathbf{D}=D \times(0, H)$ un dominio acotado tridimensional (conexo y simplemente conexo) con frontera abierta $\partial \mathbf{D}=S_{0} \cup S \cup S_{H}$, la cual es la unión de la superficie lateral cilíndrica $S$, la base $S_{0}$ en el fondo y la cubierta $S_{H}$ en $z=H$ (ver Fig. 3). Se 
denota por $\mathbf{r}_{i}=\left(x_{i}, y_{i}, Z_{i}\right) \in \mathbf{D}, i=1, \ldots, N$, los puntos donde se ubican las $N$ fuentes puntuales (fuentes industriales) que emiten $K$ especies contaminantes pasivas con tasas respectivas $q_{i}(t), i=1, \ldots, N$. Se denota con $\phi_{k}(\mathbf{r}, t)$ a la concentración del $k$-ésimo contaminante dentro de la región $\mathbf{D}$ en el punto $\mathbf{r}$ al tiempo $t$. Se supone que la región $\mathbf{D}$ contiene a todas las fuentes de emisión de los contaminantes, y por lo tanto, no habrá contribuciones externas a la contaminación dentro del dominio D. Así, la propagación de los contaminantes en la atmósfera se describe a través del siguiente sistema diferencial con parámetros continuos (ver Fig. 3):

$$
\begin{aligned}
& \left(\phi_{k}\right)_{t}+A \phi_{k}=\left(\phi_{k}\right)_{t}+\mathbf{U} \cdot \nabla \phi_{k}+\nabla \cdot \phi_{k s}-\nabla \cdot\left(\mu \nabla \phi_{k}\right)-\left[v\left(\phi_{k}\right)_{z}\right]_{z}+\sigma_{k} \phi_{k}=f_{k}(\mathbf{r}, t) \text { en } \mathbf{D} \times(0, T) \\
& \phi_{k}(\mathbf{r}, 0)=\phi_{k 0}(\mathbf{r}) \text { en } \mathbf{D} \\
& \phi_{k s}=-v_{k s} \phi_{k} \mathbf{e}_{3} \text { en } \mathbf{D} \\
& v\left(\phi_{k}\right)_{z}-U_{n} \phi_{k}=-v_{k s} \phi_{k} \text { en } S_{H^{-}}, \quad v\left(\phi_{k}\right)_{z}=-v_{k s} \phi_{k} \text { en } S_{H^{+}} \\
& \mu \nabla \phi_{k} \cdot \mathbf{n}-U_{n} \phi_{k}=0 \text { en } S^{-}, \quad \mu \nabla \phi_{k} \cdot \mathbf{n}=0 \text { en } S^{+} \\
& \mu_{d} \nabla \phi_{k} \cdot \mathbf{n}=0 \text { en } S_{0} \\
& \nabla \cdot \mathbf{U}=u_{x}+v_{y}+w_{z}=0 \text { en } \mathbf{D}
\end{aligned}
$$

Se supone que la velocidad del viento $\mathbf{U}(\mathbf{r}, t)=(u, v, w)$ en $\mathbf{D}$ cumple la ecuación de continuidad (11); $\sigma_{k}=$ $\sigma_{k}(\mathbf{r}, t) \geq 0$ es el coeficiente de transformación química de la $k$-ésima especie contaminante, $\mu_{d}(\mathbf{r}, t)=\operatorname{diag}\{\mu, \mu, v\}>0$ es el tensor diagonal de difusión turbulenta y $f_{k}(\mathbf{r}, t)$ es el forzamiento definido por las tasas de emisión para el k-ésimo contaminante:

$$
f_{k}(\mathbf{r}, t)=\sum_{i=1}^{N} q_{i k}(t) \delta\left(\mathbf{r}-\mathbf{r}_{i}\right)
$$

donde $\delta\left(\mathbf{r}-\mathbf{r}_{i}\right)$ es la función delta de Dirac centrada en la posición de la i-ésima fuente puntual. Notemos que la tasa de emisión de cada fuente es la suma de las tasas para cada contaminante, es decir,

$$
q_{i}(t)=\sum_{k=1}^{k} q_{i k}(t)
$$

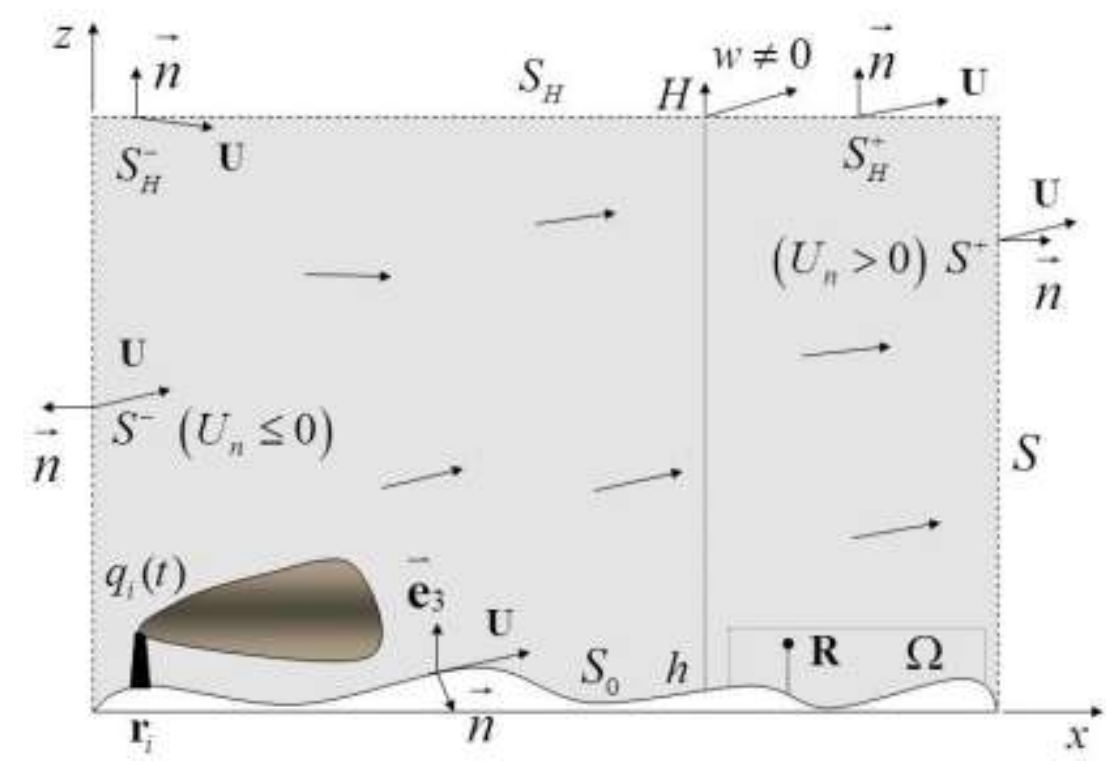

Fig. 3: Proyección en el plano x-z de la región $\mathbf{D}$.

La ecuación (6) define a $\phi_{k 0}$ como la distribución espacial de la $k$-ésima especie contaminante al tiempo $t=0$ sobre D. El término $\nabla \cdot \phi_{k s}$ en (5), describe el cambio en la concentración de las partículas por unidad de tiempo debido a la sedimentación, la cual está caracterizada por una velocidad constante de sedimentación $v_{k s}$. La frontera $S$ se ha dividido en cinco partes, dos para el flujo horizontal, es decir, $S^{+}$se define como los puntos de $S$ tal que $U_{n}=\mathbf{U} \cdot \mathbf{n}>0$, donde $\mathbf{n}$ es el vector normal exterior y $S$ se define como el complemento 
$\left(U_{n}=\mathbf{U} \cdot \mathbf{n} \leq 0\right)$. Dos secciones para la frontera superior: $S_{H^{-}}$es la frontera donde la sedimentación es el resultado de la difusión menos la advección, y $S_{H^{+}}$implica que la sedimentación es igual a la difusión (Ec. 8). Por último, en $S_{0}$, que es la frontera inferior, se tiene que la difusión es cero debido a que el flujo es tangente a la superficie irregular (Ec. 10). Las condiciones de frontera (9) establecen que cuando el viento ingresa a la región $\mathbf{D}$ el flujo total del contaminante, tomando en cuenta difusión y advección, es igual a cero, por lo cual, en $S^{-}$no hay salida o entrada de la especie contaminante. Cuando el viento sale de la región $\mathbf{D}$, se desprecia el flujo difusivo turbulento en comparación con el flujo advectivo del contaminante, por lo tanto, la salida de la especie contaminante sólo es por advección. Las condiciones de frontera (8)-(10) hacen del modelo de dispersión (5)-(11) un problema bien formulado en el sentido de Hadamard, es decir, la solución de dicho problema es única y estable respecto de pequeñas perturbaciones en las condiciones iniciales y del forzamiento (Skiba y Parra-Guevara, 2000). Este resultado es consecuencia de que el operador $A$, en la ecuación (5), es positivo semidefinido: $\left(A \phi_{k}, \phi_{k}\right) \geq 0$, donde $(\phi, \eta)=\int_{D} \phi \eta d r$ es el producto interior. De aquí se sigue la estimación que establece la unicidad y la estabilidad de la solución del modelo (5)-(11):

$$
\left\|\phi_{k}\right\|_{2} \leq T \cdot \max _{0 \leq t \leq T}\left\|f_{k}(\mathbf{r}, t)\right\|_{2}+\left\|\phi_{k}^{0}\right\|_{2}, \quad \text { donde } \quad\|\varphi\|_{2}=\left(\int_{\mathbf{D}} \varphi^{2} d \mathbf{r}\right)^{1 / 2}
$$

La existencia de la solución del problema (5)-(11) se prueba también en Skiba y Parra-Guevara (2000).

\section{ECUACIÓN DE BALANCE DE MASA Y ANÁLISIS DE EXISTENCIA}

Las condiciones de frontera (8)-(10) tienen también un adecuado sentido físico, ya que el modelo satisface una ecuación de balance de masa consistente con el fenómeno de dispersión y transformación de sustancias en la atmósfera. Para obtener tal relación se integra la ecuación (5) en el dominio D y se simplifica usando las ecuaciones (8)-(11), así obtenemos:

$$
\frac{\partial}{\partial t} \int_{\mathbf{D}} \phi_{k} d \mathbf{r}=\sum_{i=1}^{N} q_{i k}(t)-\int_{\mathbf{D}} \sigma_{k} \phi_{k} d \mathbf{r}-\int_{S_{0}} v_{k s} \phi_{k}\left|\mathbf{e}_{3} \cdot \mathbf{n}\right| d S-\int_{S^{+} \cup S_{H^{+}}} U_{n} \phi_{k} d S
$$

Esta ecuación indica que la razón de cambio de la masa total de la k-ésima especie contaminante en el dominio D, es igual a la suma de las tasas con que se emite dicha sustancia, menos la tasa de disminución por transformación química, menos la tasa de sedimentación en la frontera inferior $S_{0}$, menos la razón de pérdida de masa del contaminante que escapa del dominio $\mathbf{D}$ a través de la frontera abierta, esto último debido a la advección (notar que $U_{n} \geq 0$ en $S^{+} \cup S_{H}^{+}$).

Para el análisis de existencia de soluciones en el problema inverso se considera una fuente puntual ubicada en el sitio $\mathbf{r}_{0} \in \mathbf{D}$ que emite un contaminante con tasa $Q(t)$, y se asume que el proceso de dispersión de dicha sustancia en la atmósfera se describe por el modelo (5)-(11), por lo tanto, se satisface la ecuación de balance de masa (15) para la tasa de emisión $Q(t)$ y la concentración (de la anomalía) $\phi(\mathbf{r}, t)$. En lo sucesivo está relación se conoce como ecuación $M$. Se denota por $\mathbf{R}$ el sitio de monitoreo del contaminante en el dominio $\mathbf{D}(\mathbf{R} \notin \partial \mathbf{D})$. Además, suponemos que para cada instante $t>0$ se cumple que el coeficiente de transformación química es acotado, $|\sigma(\mathbf{r}, t)| \leq \sigma_{0}$ en $\mathbf{D}$.

Se asume ahora que la concentración del contaminante se conoce en forma exacta salvo por una pequeña perturbación en un entorno del sitio de monitoreo, es decir,

$$
\phi_{\varepsilon}(\mathbf{r}, t)=\phi(\mathbf{r}, t)+\varepsilon(t) h(\mathbf{r}), \quad \mathbf{r} \in \mathbf{D}, \quad t>0
$$

donde $\varepsilon(t)$ es una función con derivada continua tal que $|\varepsilon(t)|<<1$ para todo $t>0$, y $h(\mathbf{r})$ es la siguiente función de colocación:

$$
h(\mathbf{r})= \begin{cases}\left(1-\rho^{-2}\|\mathbf{r}-\mathbf{R}\|^{2}\right)^{2}, & \|\mathbf{r}-\mathbf{R}\| \leq \rho \\ 0, & \|\mathbf{r}-\mathbf{R}\|>\rho\end{cases}
$$

en la cual el parámetro positivo $\rho$ se elige suficientemente pequeño para satisfacer que $\left\|\mathbf{r}_{0}-\mathbf{R}\right\|>\rho$ y que $\|\mathbf{r}-\mathbf{R}\|>\rho$ para todo $\mathbf{r} \in \partial \mathbf{D}$. Note que la función no negativa $h(\mathbf{r})$ tiene derivada continua, $h(\mathbf{r}) \leq 1$ y $h(\mathbf{R})=1$. Así, $\phi(\mathbf{r}, t)$ coincide con $\phi_{\varepsilon}(\mathbf{r}, t)$ cuando $\|\mathbf{r}-\mathbf{R}\| \geq \rho$ y $t>0 ;$ y $\left|\phi_{\varepsilon}(\mathbf{r}, t)-\phi(\mathbf{r}, t)\right| \leq|\varepsilon(t)|<<1$ cuando $\|\mathbf{r}-\mathbf{R}\|<\rho$ y $t>0$. Ya que $\rho$ y $\varepsilon(t)$ se pueden elegir tan pequeños como se quiera entonces se concluye que $\phi_{\varepsilon}(\mathbf{r}, t)$ es una aproximación suave y arbitrariamente cercana a los valores exactos de la concentración. 
Supongamos que existe una tasa de emisión $Q_{\varepsilon}(t)$ que satisface el modelo (5)-(11) y reproduce los datos de concentración $\phi_{\varepsilon}(\mathbf{r}, t)$. Tal función satisfacen también la ecuación de balance de masa (15), dicha ecuación específica se denota como $M_{\varepsilon}$.

La resta de ecuaciones de balance de masa $M_{\varepsilon}-M$ proporciona la siguiente relación:

$\frac{\partial}{\partial t} \int_{D} \varepsilon(t) h(\mathbf{r}) d \mathbf{r}=Q_{\varepsilon}(t)-Q(t)-\int_{D} \sigma(\mathbf{r}, t) \varepsilon(t) h(\mathbf{r}) d \mathbf{r}$

donde las integrales en las partes de la frontera $S_{0}$ y $S^{+} \cup S_{H^{+}}$desaparecen porque $\phi_{\varepsilon}(\mathbf{r}, t)=\phi(\mathbf{r}, t)$ para toda $\mathbf{r} \in \partial \mathbf{D}$.

Al introducir la notación $\delta Q(t)=Q_{\varepsilon}(t)-Q(t)$, para el error en la tasa de emisión, se obtiene de (18) que

$\delta Q(t)=\frac{\partial}{\partial t} \varepsilon(t) \int_{\|r-R\| \leq \rho} h(\mathbf{r}) d \mathbf{r}+\varepsilon(t) \int_{\|r-R\| \leq \rho} \sigma(\mathbf{r}, t) h(\mathbf{r}) d \mathbf{r}$

donde las integrales en la ecuación (19) son positivas y están acotadas.

Ahora se considera que $\varepsilon(t)=\varepsilon 0 \operatorname{sen}(4 \omega \pi t / T)$, donde $\varepsilon_{0}>0$ es la amplitud de la perturbación $\left(\varepsilon_{0}<<1\right)$, y el número entero positivo $\omega$ es la frecuencia. Sustituyendo $\varepsilon(t)$ en la ecuación (19) se obtiene que

$\delta Q(t)=\varepsilon_{0}\left\{(4 \omega \pi / T) \cos (4 \omega \pi t / T) \int_{\|r-R\| \leq \rho} h(\mathbf{r}) d \mathbf{r}+\operatorname{sen}(4 \omega \pi t / T) \int_{\|r-R\| \leq \rho} \sigma(\mathbf{r}, t) h(\mathbf{r}) d \mathbf{r}\right\}$

Observamos que si $t=T / 2$ en la ecuación (20) entonces se tiene el siguiente resultado fundamental:

$$
\delta Q(T / 2)=\varepsilon_{0}(4 \omega \pi / T) \cos (2 \omega \pi) \int_{\|r-R\| \leq \rho} h(\mathbf{r}) d \mathbf{r}=\varepsilon_{0}(4 \omega \pi / T) \int_{\|r-R\| \leq \rho} h(\mathbf{r}) d \mathbf{r}, \quad \omega=1,2, \ldots
$$

y por lo tanto, $\delta Q(T / 2) \rightarrow \infty$ cuando $\omega \rightarrow \infty$. Además, ya que el lado derecho de la ecuación (21) es positivo entonces para un valor grande de la frecuencia $\omega$ se tiene que

$$
Q_{\varepsilon}(T / 2)>>Q(T / 2)
$$

Esto significa que en el instante $t=T / 2$ hay una emisión del contaminante en el sitio $\mathbf{r}_{0}$ mucho mayor que la esperada por la tasa $Q$, y ya que la dispersión en la atmósfera no es instantánea sino paulatina y continua, entonces la concentración $\phi_{\varepsilon}$ en un entorno del punto $\mathbf{r}_{0}$ debe ser mayor que $\phi$ en dicho instante de tiempo (e instantes posteriores). Esto contradice que $\phi_{\varepsilon}(\mathbf{r}, t)=\phi(\mathbf{r}, t)$ cuando $\|\mathbf{r}-\mathbf{R}\| \geq \rho$ y $t>0$. Por lo tanto, se concluye que dicha función $Q_{\varepsilon}(t)$ no existe.

Este resultado indica que tratar de estimar (sin una regularización del problema inverso) las tasas de emisión de fuentes y sumideros de sustancias que se propagan en la atmósfera puede resultar imposible aun considerando datos de concentración casi perfectos. Como se explica en la siguiente sección, este problema de existencia se debe al gran volumen de información utilizada (ver ecuación 16).

\section{ANÁLISIS DE ESTABILIDAD DEL PROBLEMA INVERSO}

Se considera ahora un modelo de dispersión general para una fuente puntual que emite un contaminante:

$$
(\phi)_{t}+A \phi=Q(t) \delta\left(\mathbf{r}-\mathbf{r}_{0}\right) \text { en } \mathbf{D} \times(0, T) ; \quad \phi(\mathbf{r}, 0)=0 \text { en } \mathbf{D}
$$

donde el operador lineal $A$ contiene los procesos físicos y químicos a los cuales está sometida dicha sustancia en la atmósfera y $\phi(\mathbf{r}, t)$ representa la concentración de la anomalía. Para este modelo general se considera también el correspondiente modelo adjunto (Parra-Guevara et al., 2015).

$$
-(g)_{t}+A^{*} g=p(\mathbf{r}, t) \text { en } \mathbf{D} \times(0, T) ; \quad g(\mathbf{r}, T)=0 \text { en } \mathbf{D}
$$


donde los operadores $A$ y $A^{*}$ satisfacen la identidad de Lagrange (Marchuk, 1986): $(A \phi, g)=\left(\phi, A^{*} g\right)$, y $(\phi, \psi)=\int_{\mathbf{D}} \phi \psi \mathrm{dr}$ es el producto interior en el espacio de Hilbert $L_{2}(\mathbf{D})$.

Como un caso particular del modelo definido por (24) se presenta el siguiente sistema de ecuaciones como el modelo adjunto que corresponde al modelo de dispersión (5)-(11) (Parra-Guevara et al., 2015).

$-(g)_{t}+A^{*} g=-\left(g_{k}\right)_{t}-\mathbf{U} \cdot \nabla g_{k}-\nabla \cdot g_{k s}-\nabla \cdot\left(\mu \nabla g_{k}\right)-\left[\nu\left(g_{k}\right)_{z}\right]_{z}+\sigma_{k} g_{k}=p(\mathbf{r}, t)$

$g_{k}(\mathbf{r}, T)=0$ en $\mathbf{D}$

$g_{k s}=-v_{k s} g_{k} \mathbf{e}_{3}$ en $\mathbf{D}$

$v\left(g_{k}\right)_{z}=0$ en $S_{H^{-}}, \quad v\left(g_{k}\right)_{z}+U_{n} g_{k}=0$ en $S_{H^{+}}$

$\mu \nabla g_{k} \cdot \mathbf{n}=0 \quad$ en $S^{-}, \quad \mu \nabla g_{k} \cdot \mathbf{n}+U_{n} g_{k}=0 \quad$ en $S^{+}$

$\mu_{d} \nabla g_{k} \cdot \mathbf{n}+g_{k s} \cdot \mathbf{n}=0$ en $S_{0}$

Es importante notar que el modelo (25)-(30) es un problema de valor final en donde la velocidad del flujo y la sedimentación han invertido su dirección, además, el cambio de variable $t^{\prime}=T-$ - $t$ lo convierte en un problema de valor inicial bien formulado, la demostración es similar a la del problema (5)-(11).

Cuando el forzamiento en la ecuación general (24) se elige como $p(\mathbf{r}, t)=\delta(\mathbf{r}-\mathbf{R}) \delta(t-\tau)$, para un instante arbitrario $\tau$, es posible obtener el principio de dualidad (ecuación integral) que relaciona las concentraciones puntuales del contaminante con la tasa de emisión $Q(t)$ de la fuente puntual (Parra-Guevara et al., 2015).

$\phi(\mathbf{R}, \tau)=\int_{0}^{T} Q(t) g\left(\mathbf{r}_{0}, t ; \tau\right) d t$

donde la función adjunta $g(\mathbf{r}, t ; \tau)$ es la solución del modelo (24) con el forzamiento $p(\mathbf{r}, t)=\delta(\mathbf{r}-\mathbf{R}) \delta(t-\tau)$. La Figura 4 muestra ejemplos de soluciones adjuntas $g_{\tau}=g\left(\mathbf{r}_{0}, t ; \tau\right)$ de la versión $1 \mathrm{D}$ del modelo (25)-(30).

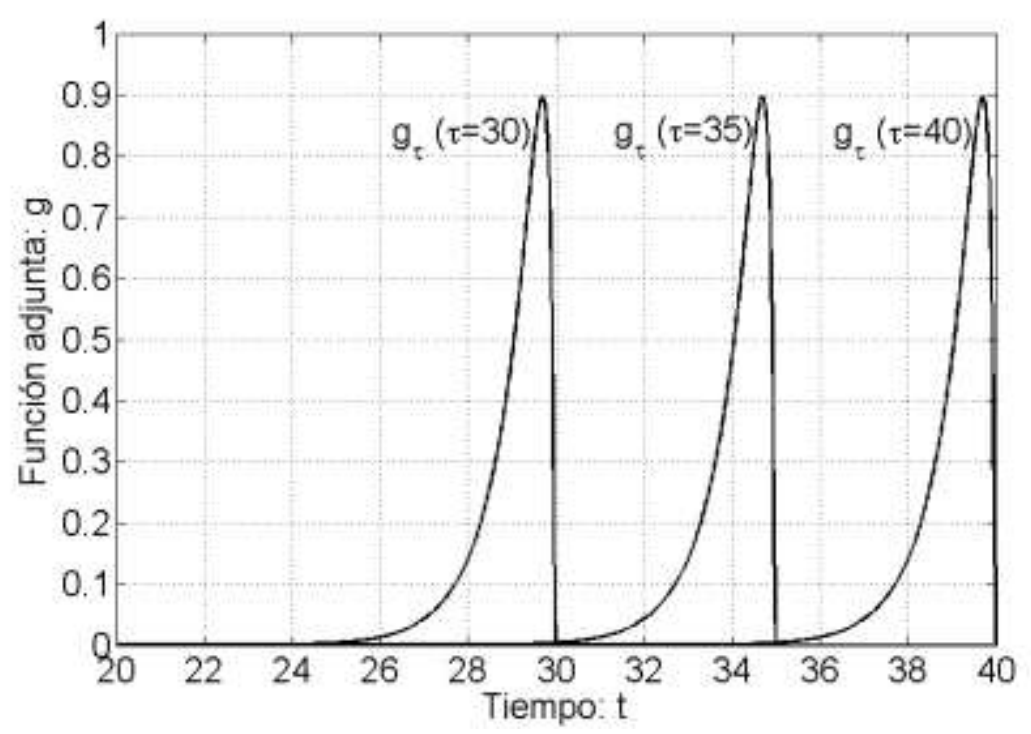

Fig. 4: Ejemplos de funciones adjuntas $g_{\tau}=g\left(\mathbf{r}_{0}, t ; \tau\right)$.

Se considera ahora una pequeña perturbación (continua) $\varepsilon(\tau)$ en los datos puntuales de la concentración:

$\phi_{\varepsilon}(\mathbf{R}, \tau)=\phi(\mathbf{R}, \tau)+\varepsilon(\tau), \quad \tau>0$

Note que la ecuación (32) involucra un volumen mucho menor de datos que los requeridos en la Ec. 16. Denotamos por $Q_{\varepsilon}(t)$ la tasa de emisión que satisface la ecuación (31) para dichos datos $\phi_{\varepsilon}(\mathbf{R}, \tau)$, es decir, 
$\phi_{\varepsilon}(\mathbf{R}, \tau)=\int_{0}^{T} Q_{\varepsilon}(t) g\left(\mathbf{r}_{0}, t ; \tau\right) d t$

Restando las ecuaciones (33) y (31), y considerando la relación (32), se obtiene que

$$
\varepsilon(\tau)=\int_{0}^{T} \delta Q(t) g\left(\mathbf{r}_{0}, t ; \tau\right) d t ; \quad \delta Q(t)=Q_{\varepsilon}(t)-Q(t)
$$

Derivando respecto del parámetro $\tau$ en ambos lados de la ecuación integral (34), y considerando la desigualdad de Schwarz en el espacio de Hilbert $L_{2}(0, T)$, se obtiene la siguiente estimación:

$$
\left|\partial_{\tau}\{\varepsilon(\tau)\}\right| \leq\|\delta Q(t)\|\left\|\partial_{\tau}\left\{g\left(\mathbf{r}_{0}, t ; \tau\right)\right\}\right\|
$$

Teorema 1. Si $\left\|\partial_{\tau}\left\{g\left(\mathbf{r}_{0}, t ; \tau\right)\right\}\right\| \leq C, \tau>0$, entonces hay inestabilidad en la recuperación de la tasa de emisión. Demostración. Sea $\varepsilon(\tau)=\varepsilon_{0} \operatorname{sen}(4 \omega \pi \tau / T)$, donde $\varepsilon_{0}>0$ es la amplitud de la perturbación $\left(\varepsilon_{0}<<1\right)$. Sustituyendo $\varepsilon(\tau)$ en (35) se obtiene la siguiente estimación: $\varepsilon 0(4 \omega \pi / T C)|\cos (4 \omega \pi \tau / T)| \leq\|\delta Q(t)\|$. Si $\tau=T / 2$ en tal relación entonces se tiene que $\varepsilon_{0} \omega(4 \pi T C) \leq\|\delta Q(t)\|, \omega=1,2, \ldots$, y por lo tanto, $\|\delta Q(t)\| \rightarrow \infty$ cuando $\omega \rightarrow \infty$. De esta forma, aún cuando $\varepsilon 0<<1$, el error en la estimación de la tasa de emisión $\delta Q(t)$ es arbitrariamente grande cuando la frecuencia de la perturbación se incrementa. Esto prueba la inestabilidad.

La desigualdad $\varepsilon_{0} \omega(4 \pi / T C) \leq\|\delta Q(t)\|$ muestra que el error en la estimación de la tasa de emisión $\delta Q(t)$ crece sobre un intervalo finito $[0, T]$ en forma proporcional al producto de la amplitud $\varepsilon 0$ y la frecuencia $\omega$ de la perturbación en los datos. Ya que el aumento de la frecuencia puede empeorar la estimación de la tasa de emisión, esto debería prevenir el intento de recuperar dicha función sólo con el incremento del número de datos en el intervalo $[0,7]$, es decir, incrementando la frecuencia de muestreo. De aquí se concluye que es indispensable introducir una regularización para resolver el problema inverso. Tal regularización debe filtrar dichos errores de alta frecuencia en los datos (Parra-Guevara et al., 2015).

Sobre la existencia y la unicidad de soluciones de la ecuación integral

Para mostrar la existencia de una solución $Q_{\varepsilon}(t)$ de la ecuación (33) consideramos la condición general $g\left(\mathbf{r}_{0}, t ; \tau\right)=0$ para $t \geq \tau$ (Parra-Guevara et al., 2015), junto con condiciones de dispersión estacionarias en la atmósfera. De esta forma es posible obtener de (33) la siguiente ecuación de Volterra de primer orden:

$$
\phi_{\varepsilon}(\mathbf{R}, \tau)=\int_{0}^{\tau} Q_{\varepsilon}(t) g_{\alpha}\left(\mathbf{r}_{0}, T+t-\tau\right) d t
$$

donde $g_{\alpha}$ es la función adjunta para $\tau=T$, llamada núcleo básico, y las funciones adjuntas $g\left(\mathbf{r}_{0}, t ; \tau\right)$ se generan a través de translaciones de dicha función, es decir, $g\left(\mathbf{r}_{0}, t ; \tau\right)=g_{\alpha}\left(\mathbf{r}_{0}, T+t-\tau\right)$ en $(0, \tau)$ (Parra-Guevara et al., 2015). Si se define la función $G(\xi)=g_{\alpha}\left(\mathbf{r}_{0}, T-\xi\right)$ entonces la ecuación integral (36) se escribe como una ecuación de convolución (Rahman, 2007):

$$
\phi_{\varepsilon}(\mathbf{R}, \tau)=\int_{0}^{\tau} Q_{\varepsilon}(t) G(\tau-t) d t=\left(Q_{\varepsilon} * G\right)(\tau)
$$

Resolviendo la ecuación (37) por el método de la transformada de Laplace (Rahman, 2007), se obtiene:

$$
L_{a}\left\{\phi_{\varepsilon}\right\}=L_{a}\left\{Q_{\varepsilon} * G\right\}=L_{a}\left\{Q_{\varepsilon}\right\} L_{a}\{G\}
$$

donde $L_{a}\{\cdot\}$ denota la transformada de Laplace. Arreglando términos en la ecuación (38) se concluye que

$$
Q_{\varepsilon}(\tau)=\left(\phi_{\varepsilon} * \psi\right)(\tau)=\int_{0}^{\tau} \phi_{\varepsilon}(t) \psi(\tau-t) d t, \quad \psi(\xi)=L_{a}^{-1}\left\{1 / L_{a}\{G(\xi)\}\right\}
$$

Note que la solución $Q_{\varepsilon}(\tau)$ está bien definida porque las funciones $\phi_{\varepsilon}$ y $G$ son continuas y acotadas, lo que implica que tanto las transformadas del lado derecho en la definición de la función $\psi(\xi)$ como la integral de convolución en (39) existen.

Para mostrar que la tasa de emisión $Q_{\varepsilon}$ que reproduce los datos $\phi_{\varepsilon}$ no es única basta notar que la ecuación integral (33) admite más de una solución en el intervalo $[0,7]$. Esto es consecuencia de que existe un intervalo abierto $(\eta, T)$ tal que 


$$
g\left(\mathbf{r}_{0}, t ; \tau\right)=0, \quad \forall \tau \in[0, T] \quad \text { y } \quad \forall t \in(\eta, T)
$$

y por lo tanto, la función $Q_{\varepsilon}(t)$ puede tomar cualesquiera valores sobre dicho intervalo sin alterar la ecuación integral. Tal característica permite generar una infinidad de soluciones a partir de una conocida. La longitud de dicho intervalo, $\Delta=T-\eta>0$, es el tiempo de transporte en la atmósfera que a cualquier sustancia emitida desde el sitio $\mathbf{r}_{0}$ le toma para alcanzar al sitio de monitoreo $\mathbf{R}$.

Este resultado implica que a través de los datos de concentración recabados en el sitio $\mathbf{R}$, es decir, la serie de tiempo $\left\{\phi_{j}, j=0,1, \ldots, L\right\}$ en el intervalo $[0, T]$, es imposible obtener la tasa real de emisión $Q(t)$ en el intervalo $(\eta, T)$. Esto es así porque la masa emitida durante dicho intervalo final todavía no ha sido registrada en el instrumento de medición.

\section{REGULARIZACIÓN DEL PROBLEMA INVERSO}

La ecuación (39) permite calcular la tasa de emisión de una fuente puntual a partir de la convolución de los datos de concentración con la función $\psi$, la cual sólo depende del proceso de transporte en la atmósfera. Sin embargo, como se demostró en la sección anterior, dicho cálculo es inestable cuando los datos de concentración contienen una perturbación de alta frecuencia, de aquí que resulte necesario regularizar el problema inverso para recuperar correctamente la tasa de emisión. La regularización que se sugiere se formula como un problema variacional:

$$
\begin{array}{ll}
\text { minimizar } & J(q)=\frac{1}{2} \int_{0}^{T}\left[q^{\prime}(t)\right]^{2} d t \\
\text { sujeto a: } & -\varepsilon \leq \phi_{j}-\int_{0}^{t_{j}} q(t) g\left(\mathbf{r}_{0}, t ; \tau_{j}\right) d t \leq \varepsilon, \quad j=1, \ldots, M
\end{array}
$$

donde $q(t) \geq 0$ en $(0, T)$ y $q(0)=q(T)=0$. Además, $\phi_{F}=\phi\left(\mathbf{R}, t_{j}\right)+\delta \phi_{j}$, y $\delta \phi_{j}$ es el error para la anomalía de la concentración del contaminante en el instante $t=t j, j=1, \ldots, M$. La solución regularizada $Q_{\varepsilon}$ del problema inverso es precisamente la solución del problema variacional (41).

El funcional $J(q)$ en (41) se minimiza para filtrar las perturbaciones y reconstruir la tasa de emisión. Además, se considera la diferencia de términos en las restricciones en (41) porque debido a los errores $\delta \phi_{j}$ es imposible utilizar directamente la ecuación (31). El parámetro de regularización $\varepsilon>0$ se introduce para extender el espacio factible de forma que exista una solución del problema variacional, y por lo tanto del problema inverso. Este parámetro influye en la suavidad de la solución regularizada $Q_{\varepsilon}$, y su valor óptimo, determinado a través de experimentos numéricos, es $\varepsilon^{*}=\max _{j}\left|\delta \phi_{j}\right|$ (Parra-Guevara et al., 2015). En ParraGuevara et al. (2015) se prueba la existencia y la unicidad de la solución del problema variacional (41). Dicha solución se obtiene en forma numérica al resolver un problema de programación cuadrática, el cual es la versión discreta del problema (41) en las variables $q_{i}, i=1, \ldots, M$ (Parra-Guevara et al., 2015). En los experimentos numéricos se utiliza la rutina quadprog de MATLAB (Venkataraman, 2002) para resolver dicho problema de optimización en forma eficiente.

Ejemplos. Consideramos ahora la versión unidimensional de los modelos (5)-(11) y (25)-(30), en el dominio $\mathbf{D}=[0,1]$ e intervalo $[0, T](T=40)$, con los siguientes parámetros adimensionales: $\mathbf{U}=0.85 \mathbf{e}_{1}, v_{k s}=0, \mu=0.5, v=0$, $\sigma=0.1, \mathbf{r}_{0}=0.3$ y $\mathbf{R}=0.8$. Además, se considera la siguiente función de prueba: $Q(t)=100 t^{2} \mathrm{e}^{-0.5 t} \operatorname{sen}^{2}(t), 0 \leq t \leq 4 \pi$; $Q(t)=0,4 \pi \leq t \leq 40$. En la Figura 5 se muestra la gráfica de la función $Q(t)$, así como la gráfica de la solución del modelo unidimensional de dispersión en $x=\mathbf{R}$, es decir, $\phi(\mathbf{R}, \mathrm{t})$. La gráfica del núcleo básico $g_{\alpha}(\tau=40)$ y dos ejemplos de funciones adjuntas ( $\tau=30$ y 35 ) se muestran en la Figura 4 . Como fue explicado, cada función adjunta es una translación en el tiempo del núcleo básico. Una vez calculadas las funciones adjuntas es posible plantear y resolver el problema de programación cuadrática, y por lo tanto, obtener la solución regularizada del problema inverso. Para los experimentos numéricos la serie de tiempo sintética $\left\{\phi_{j}, j=0, \ldots, M\right\}$, es generada como $\phi_{F} \phi_{j}\left(\mathbf{R}, t_{j}\right)+\delta \phi_{j}$, donde $\delta \phi_{j}$ son valores aleatorios uniformemente distribuidos en el intervalo $(-0.5,0.5)$. La amplitud del error fue normalizada para no exceder el $15 \%$ del valor máximo de la función $\phi(\mathbf{R}, t)$. La Figura 6 presenta la función $Q(t)$ y el comportamiento de las soluciones regularizadas cuando $\varepsilon$ se aproxima a $\varepsilon^{*}$ (desde la derecha), donde $\varepsilon=e \varepsilon^{*}, \varepsilon^{*}=\max _{j}\left|\delta \phi_{j}\right|$ y $e=2.0,1.6$ y 1.002 . Los experimentos numéricos muestran que la solución del problema variacional (41) se suaviza y tiende a la función cero cuando $\varepsilon \rightarrow \infty$ (ya que el espacio factible del problema (41) se expande hasta contener el mínimo global del funcional objetivo). Además, para $\varepsilon=\varepsilon^{*}$ se obtiene la mejor aproximación de la tasa $Q(t)$ que esta regularización puede proveer. 


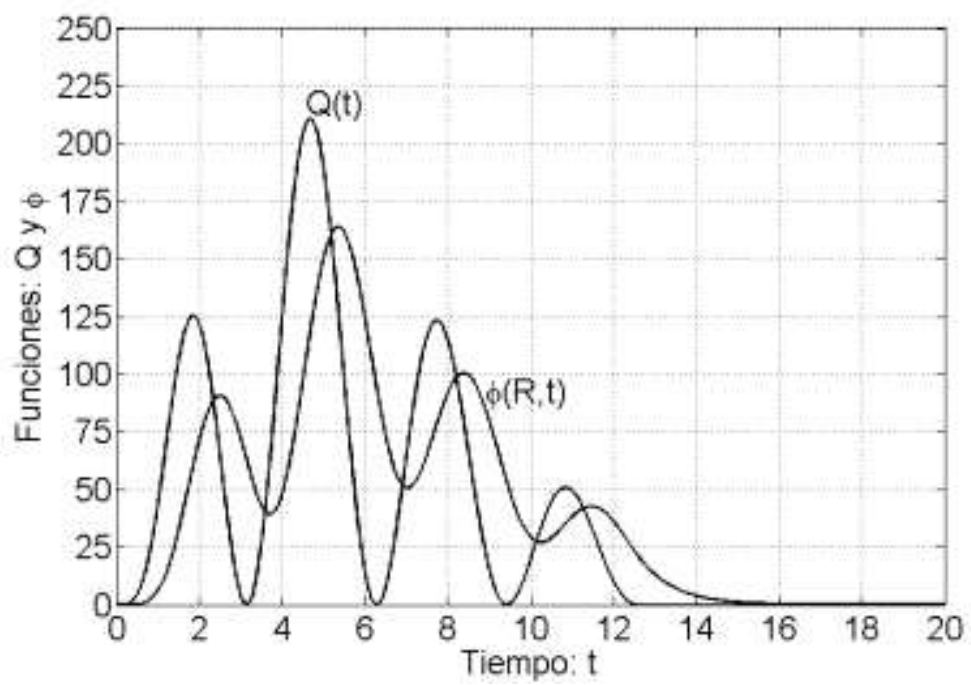

Fig. 5: Tasa de emisión $Q(t)$ y concentración $\phi$ en el sitio de monitoreo $x=\mathbf{R}$.

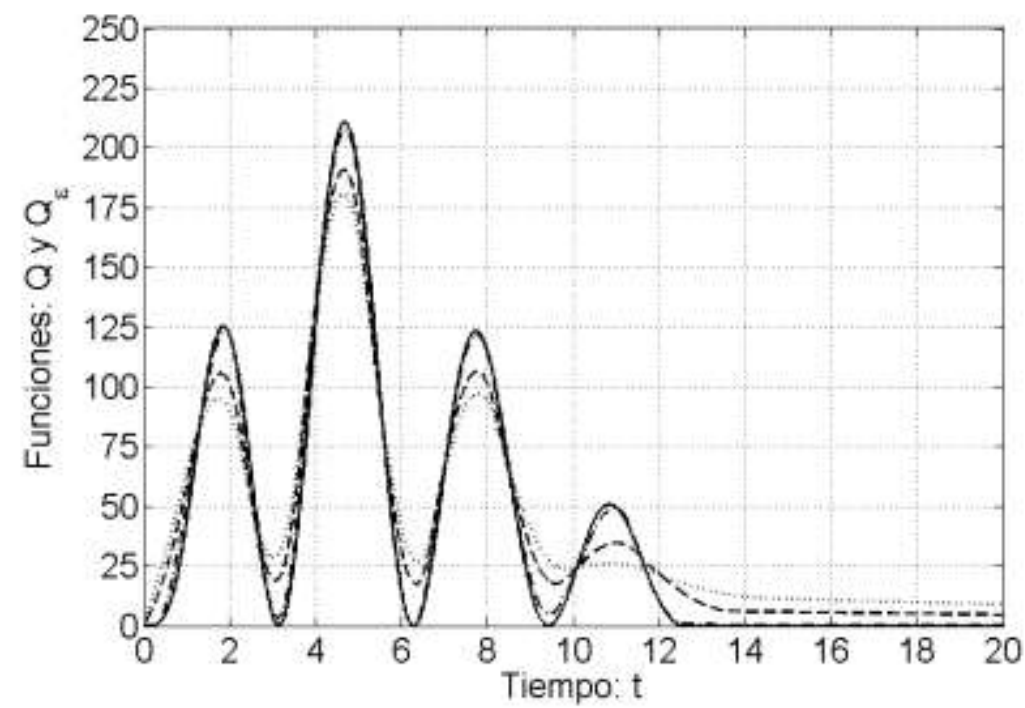

Fig. 6: Función $Q$ (línea continua), y comportamiento de la solución regularizada $Q_{\varepsilon}$ cuando $\varepsilon \rightarrow \varepsilon^{*}$ : $e=2.000$, línea punteada (.....); $e=1.600$, línea raya-espacio (-_-_-); y $e=1.002$, línea punto-raya (.---_-).

\section{CONCLUSIONES}

El análisis realizado muestra que la recuperación de la tasa de emisión de una fuente puntual noestacionaria a partir de datos de la concentración de contaminantes es un problema inverso inestable, con dificultades en los aspectos de la existencia y la unicidad de las soluciones. A este respecto, las conclusiones específicas del estudio son las siguientes: 1) este problema inverso es particularmente sensible a las perturbaciones de alta frecuencia contenidas en los datos de la concentración de los contaminantes; 2) incrementar la frecuencia de muestreo para incluir un mayor número de datos de concentración en el proceso de inversión tiende a empeorar la estimación de la tasa de emisión; 3) aumentar los sitios de monitoreo con el fin de incluir un mayor número de datos de concentración en el proceso de inversión conduce a la inexistencia de soluciones del problema inverso; 4) para obtener una correcta estimación de la tasa de emisión es indispensable introducir una regularización en el problema inverso que filtre los errores responsables de la inestabilidad; y 5 ) la regularización definida por la ecuación (41), basada en la minimización de la norma de la primera derivada de $Q$, filtra el error de alta frecuencia en los datos y permite obtener la tasa de emisión en forma adecuada.

\section{AGRADECIMIENTOS}

Este trabajo fue apoyado por el Sistema Nacional de Investigadores (CONACyT-México), becas No. 25170 y 14539, y por el proyecto No. IN101116-2, PAPIIT, DGAPA-UNAM, México. 


\section{REFERENCIAS}

Bolin, B. y C. D. Keeling, Large-scale atmospheric mixing as deduced from the seasonal and meridional variations of carbon dioxide, Journal of Geophysical Research, 68(13), 3899-3920 (1963)

Brioude, J., S.-W. Kim, W. M. Angevine, G. J. Frost, S.-H. Lee, S. A. Mckeen, M. Trainer, F. C. Fehsenfeld, J. S. Holloway, T. B. Ryerson, E. J. Williams, G. Petron y J. D. Fast, Top-down estimate of anthropogenic emission inventories and their interannual variability in Houston using a mesoscale inverse modeling technique, Journal of Geophysical Research, 116, D20305 (2011)

Enting, I. G. y G. N. Newsam, Atmospheric constituent inversion problems: Implications for baseline monitoring, Journal of Atmospheric Chemistry, 11, 69-87 (1990)

Lushi, E. y J. M. Stockie, An inverse Gaussian plume approach for estimating atmospheric pollutant emissions from multiple point sources, Atmospheric Environment, 44, 1097-1107 (2010)

Marchuk, G. I., Mathematical models in environmental problems. Elsevier, New York (1986)

Parra-Guevara, D., Y. N. Skiba y A. Reyes-Romero; Existence and uniqueness of the regularized solution in the problem of recovery of the non-steady emission rate of a point source: Application of the adjoint method. In: Proceedings of the International Conference on Engineering Optimization: Engineering Optimization IV, Rodrigues, H.C., et al. (eds.), Taylor and Francis Group, London, UK, 181-186 (2015)

Parra-Guevara, D., Y. N. Skiba y A. Pérez-Sesma, A linear programming model for controlling air pollution, International Journal of Applied Mathematics, 23(3), 549-569 (2010)

Parra-Guevara, D. y Y. N. Skiba, Elements of the mathematical modeling in the control of pollutants emissions, Ecological Modelling,167(3), 263-275 (2003)

Pudykiewicz, J. A., Application of adjoint tracer transport equations for evaluating source parameters, Atmospheric Environment: 32(17), 3039-3050 (1998)

Rahman, M., Integral equations and their applications, WIT Press, Southampton, Boston (2007)

Rojano, R., H. Arregoces, y G. Restrepo, Composición Elemental y Fuentes de Origen de Partículas Respirables (PM10) y Partículas Suspendidas Totales (PST) en el Área Urbana de la Ciudad de Riohacha, Colombia, doi: 10.4067/S0718-07642014000600002, Inf. Tecnol. (en línea), 25(6), 3-12 (2014)

Seibert P., A. Frank y H. Kromp-Kolb, Inverse modelling of atmospheric trace substances on the regional scale with Lagrangian models. Proceedings from the EUROTRAC-2 Symposium-2002, Margraf Verlag, Weikersheim: 1-6 (2002)

Sharma, L. K., A. K. Ghosh, R. N. Nair, M. Chopra, F. Sunny y V. D. Puranik, Inverse modeling for aquatic source and transport parameters identification and its application to Fukushima nuclear accident, Environmental Modeling and Assessment, 19(3), 193-206 (2014)

Skaggs, T. H. y Z. J. Kabala, Recovering the release history of a groundwater contaminant, Water Resources Research, 30(1), 71-79 (1994).

Skiba, Y. N. y D. Parra-Guevara, Industrial pollution transport. Part I: Formulation of the problem and air pollution estimates, Environmental Modeling and Assessment, 5(3), 169-175 (2000)

Vázquez, C., F. Beristain, y E. Audelo, Caracterización del Patrón de Comportamiento de las Partículas en Suspensión en el Valle de México, doi: 10.4067/S0718-07642012000300002, Inf. Tecnol. (en línea). 23(3), 3-10 (2012)

Venkataraman, P., Applied optimization with MATLAB programming. John Wiley and Sons, New York (2002) 